\title{
Comparing the socioeconomic status of critical care doctors and patients
}

\author{
Peter George Brindley, MD, FRCPC • Matthew J. Douma, MD, RN • \\ Martin Beed, FFICM, DM - Daniel Garros, MD, FRCPC
}

Received: 9 October 2017/Revised: 26 October 2017/ Accepted: 14 November 2017/Published online: 27 November 2017

(c) Canadian Anesthesiologists' Society 2017

\section{To the Editor,}

Efforts have been made to increase the diversity of medical doctors, ${ }^{1}$ including encouraging females, visible minorities, and rural residents to pursue a career in medicine. However, socioeconomic status (SES) may have a greater impact upon clinician empathy, patientadherence, and health outcome than gender, ethnicity, or geography. ${ }^{2,3}$ Trainees from lower SES may be especially under-represented in critical care medicine (CCM) or whenever medical training is very expensive, specialisttraining is lengthy, or subspecialist employment is uncertain. For these reasons, we conducted the first study comparing income and education levels for parents of CCM trainees and parents of pediatric critically ill patients.

We consented Canadian senior CCM trainees in 2010, 2011, and 2012 and reported their parents'/guardians' education levels and best employment descriptor using the Blishen index. ${ }^{4}$ We compared these against the distribution of highest educational achievement published in the 2006

P. G. Brindley, MD, FRCPC ( $)$

Department of Critical Care Medicine, Department of Anesthesiology and Pain Medicine, and the Dosseter Ethics Centre, University of Alberta, Edmonton, AB, Canada

e-mail: peter.brindley@albertahealthservices.ca

M. J. Douma, MD, RN

Faculty of Nursing, Royal Alexandra Hospital, Edmonton, AB, Canada

M. Beed, FFICM, DM

Department of Anaesthesia and Intensive Care, Nottingham University Hospitals, Nottingham, UK

D. Garros, MD, FRCPC

Division of Pediatric Critical Care, Stollery Children's Hospital, Edmonton, AB, Canada
Canadian census. ${ }^{5}$ Next, we prospectively accessed similar data on pediatric intensive care unit (ICU) patients at the University of Alberta Hospital, along with parental satisfaction scores, whereby parents rated the doctors caring for their children in terms of overall care, sympathy, and parental engagement in decision-making.

We obtained 134 complete responses from senior trainees $(89 \%)$ and 80 from pediatric ICU parents (73\%). Results are summarized in the Table. Median paternal SES was significantly higher for trainees $v s$ patients (68 vs 41; $P$ $<0.001)$. Median maternal SES score was also significantly higher (62 vs 42; $P<0.001)$. A larger proportion of CCM trainee parents had completed college, graduate, or higher education compared with pediatric parents $(>80 \%$ vs $<50 \%)$. More pediatric parents were "stay at home mothers" (60\% vs 27\%; $P=0.05)$. When compared against the 2006 Canadian census data, ${ }^{5}$ parents of CCM trainees were even less representative of the general population because of their higher average educational achievements. There was no correlation between SES and satisfaction scores from the parents of critically ill patients.

Our data-albeit with limitations-suggest overall that critical care doctors from more advantaged backgrounds look after patients from comparatively more disadvantaged backgrounds. For some this will confirm common sense; for others it may be an uncomfortable realization. Fortunately, there was no gross evidence of lower satisfaction from the parents of patients, though with the caveat that satisfaction scoring was more tied to interactions with the attending physician than with senior trainees.

We are certainly not proposing punitive redress, simply that more objective data could facilitate mature and empathic debate. Following these pilot data, we could 
Table Comparison of parental socioeconomic scores for critical care trainees and pediatric intensive care patients

\begin{tabular}{llll}
\hline & Critical care trainees & Pediatric intensive care patients & $P$ value* \\
\hline Maternal score; median [IQR] & $62[45-70]$ & $42[41-44]$ & $n=32$ \\
& $n=98$ & $401[35-41]$ \\
Paternal score; median [IQR] & $68[55-76]$ & $n=56$ & $<0.001$ \\
& $n=134$ & $41[39-45]$ \\
Highest parent score; median [IQR] & $70[59-76]$ & $n=80$ & $<0.001$ \\
& $n=134$ &
\end{tabular}

*Significance calculated using two-tailed Mann-Whitney $U$-test. IQR = interquartile range

investigate further any association between SES and the treatments offered, compliance after discharge, and eventual outcome. This is because lower SES may be associated with higher costs to the system, higher disease incidence and severity, lower compliance, and poorer prognoses. Alternatively, differences between patients and providers may be of no significant measurable consequence for patients.

Canada is an intriguing first environment in which to compare SES, given our government-sponsored health system and an expectation of equal access and opportunity. Data from other countries, specialties, and professions could inform a wider discussion. On the one hand there has been a push to influence medical graduate diversity, and on the other there appears to be growing worldwide income disparity. Accordingly, we feel it is worthwhile determining to what extent doctors resemble their patients $v s$ their parents. Above all, we ought to investigate what this means for patient care.

Ethics approval Universities of Alberta and Laval, Canada.

Disclosures/conflicts None declared.
Editorial responsibility This submission was handled by Dr. Sangeeta Mehta, Associate Editor, Canadian Journal of Anesthesia.

\section{References}

1. Ayeni B. CFMS/FEMC Position Paper - Diversity in Medicine in Canada: Building a Representative and Responsive Medical Community - Updated 2012. Available from URL: https://www. cfms.org/files/position-papers/diversity_in_medicine_-_updated_ 2010_cait_c_.pdf (accessed November 2017).

2. Willems S, De Maesschalck S, Deveugele M, Derese A, De Maeseneer J. Socio-economic status of the patient and doctorpatient communication: does it make a difference? Patient Educ Couns 2005; 56: 139-46.

3. Ferguson WJ, Candib LM. Culture, language, and the doctorpatient relationship. Fam Med 2002; 34: 353-61.

4. Blishen BR, Carroll WK, Moore C. The 1981 socioeconomic index for occupations in Canada. Can Rev Sociol 1987; 24: 465-88.

5. Statistics Canada. Population 15 years and over by highest certificate, diploma or degree, by age groups (2006 census). Available from URL: http://www.statcan.gc.ca/tables-tableaux/ sum-som/101/cst01/educ43b-eng.htm (accessed November 2017). 